\title{
Análise das interações entre dados climáticos e o processo de desertificação no núcleo de desertificação de Cabrobó-PE, Brasil
}

\author{
Analysis of the interactions between climate data and the \\ desertification process in the desertification hotspot of Cabrobó- \\ PE, Brazil
}

Rita Márcia da Silva Pinto Vieira ${ }^{a}$ Ana Paula Martins do Amaral Cunha ${ }^{b}$ Alexandre Augusto Barbosa ${ }^{c}$ Germano Gondim Ribeiro Neto ${ }^{d}$ Javier Tomasella ${ }^{\mathrm{e}}$ Regina Célia dos Santos Alvalá Fabrícia Cristina Santos ${ }^{g}$ Marcos de Oliveira Santanah
${ }^{a}$ Centro Nacional de Monitoramento e Alertas de Desastres Naturais, São José dos Campos, SP, Brasil. End. Eletrônico: ritamsp@gmail.com

${ }^{b}$ Centro Nacional de Monitoramento e Alertas de Desastres Naturais, São José dos Campos, SP, Brasil. End. Eletrônico: ana.cunha@cemaden.gov.br 'Instituto Nacional de Pesquisas Espaciais, São José dos Campos, SP, Brasil. End. Eletrônico: barbosa.ale@gmail.com

${ }^{d}$ Centro Nacional de Monitoramento e Alertas de Desastres Naturais, São José dos Campos, SP, Brasil. End. Eletrônico: germano.neto@cemaden.gov.br

${ }^{e}$ Centro Nacional de Monitoramento e Alertas de Desastres Naturais, São José dos Campos, SP, Brasil. End. Eletrônico: javier.tomasella@cemaden.gov.br

${ }^{f}$ Centro Nacional de Monitoramento e Alertas de Desastres Naturais, São José dos Campos, SP, Brasil. End. Eletrônico: regina.alvala@cemaden.gov.br

IInstituto Nacional de Pesquisas Espaciais, São José dos Campos, SP, Brasil. End. Eletrônico: fabricia.santos@inpe.br

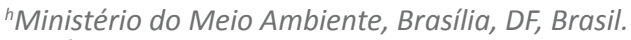
End. Eletrônico: marcos-oliveira.santana@mma.gov.br

doi:10.18472/SustDeb.v9n2.2018.27559

Recebido em 17.10.2017

Aceito em 25.05.2018

ARTIGO - VARIA 


\title{
RESUMO
}

No presente estudo avaliou-se a dinâmica das mudanças de usos e cobertura da terra no núcleo de desertificação de Cabrobó-PE, a partir de abordagem que inclui a variação espaço-temporal de dados ambientais e de clima. Para a execução do trabalho, utilizaram-se o software ArcGis e a Plataforma do Google Earth Engine. Essa plataforma combina um catálogo de imagens de satélite e conjuntos de dados geoespaciais com recursos de análise em escala planetária e os disponibiliza a cientistas, pesquisadores e desenvolvedores, o que possibilita detectar mudanças, mapear tendências e quantificar diferenças de alterações na superfície terrestre. De acordo com os resultados obtidos, foi possível constatar que as áreas de floresta no núcleo de Cabrobó diminuíram 54\%, sendo estas substituídas por áreas de solo exposto e áreas degradadas no período de 2000 a 2016. As áreas degradadas foram caracterizadas por apresentarem temperatura de superfície mais elevada $(37,5 \mathrm{oC})$, tendo aumentado em 1 oC no período de 2009 a 2016. O aumento da temperatura da superfície pode estar associado ao estresse hídrico que tende a se agravar em áreas com alto grau de degradação do solo. A combinação das informações analisadas no presente estudo permite monitorar áreas heterogêneas, identificando pontos críticos (hotspots) que requerem ações imediatas de recuperação e implementação de manejo adequado.

Palavras-chave: Degradação do Solo; Nordeste do Brasil; Mudança de Uso e Cobertura da Terra; Clima.

\begin{abstract}
This study assessed the dynamics of the Land Use and Land Cover Changes in the desertification hotspot of Cabrobó-PE using an approach that includes the space-time variation of environmental and climate data. To achieve this goal, the ArcGis software and the Google Earth Engine Platform were used. This platform combines a catalog of satellite imagery and geospatial datasets with planetary-scale analysis capabilities and makes it available for scientists, researchers, and developers allowing the detection of changes, map trends, and quantify differences on the Earth's surface. Results showed that forested areas decreased 54\% between 2000 and 2016, being replaced by areas of bare soil and degraded areas. The degraded areas were characterized by higher land surface temperature $\left(37.5 \%{ }^{\circ} \mathrm{C}\right)$ and by an increase of $1^{\circ} \mathrm{C}$ in the period 2009 to 2016. The increase in land surface temperature may be associated with the water stress, which usually worsens in areas with a high degree of soil degradation. The combination of the information analyzed in the present study allows the monitoring of heterogeneous areas, identifying hotspots that require immediate recovery actions and the improved of soil management.
\end{abstract}

Keywords: Soil degradation; Northeast of Brazil; Land Use and Land Cover Change; Climate.

\section{INTRODUÇÃO}

A A região semiárida do Brasil caracteriza-se por ser uma das mais complexas do país do ponto de vista ambiental, principalmente em razão da heterogeneidade de sua paisagem. A região é constituída por várias sub-regiões, com predomínio de grande diversificação de clima, vegetação, solo e aspectos socioeconômicos. Com relação aos usos e cobertura da terra, aproximadamente $60 \%$ da região corresponde às áreas de atividades agropecuárias e somente $15 \%$ de remanescentes da vegetação natural Caatinga (VIEIRA et al., 2013). A Caatinga é um ecossistema altamente dinâmico, que responde rapidamente às condições de clima. A distribuição da precipitação é o fator predominante que controla a estrutura e distribuição da vegetação em toda a região.

Pela heterogeneidade da sua paisagem, a região abriga uma grande riqueza de espécies, sendo a estrutura do ambiente fundamental para determinar a distribuição e diversidade destas (MACARTHUR; MACARTHUR, 1961).

Um grande desafio enfrentado pelos tomadores de decisão é como preservar essa riqueza de biodiversidade em uma área apontada como uma das mais vulneráveis às alterações climáticas para o próximo século (IPCC, 2007). A região ainda sofre com o acelerado processo de mudança de uso e cobertura da terra ocasionada pela alta densidade demográfica (34 habitantes por km2) (IBGE, 2010). 
As florestas, como sistemas naturais, prestam serviços vitais à sociedade, incluindo oportunidades econômicas e bem-estar social. A Caatinga, por exemplo, com vegetação de rara biodiversidade, vem sustentando a economia da região Nordeste por meio do fornecimento de energia, com $33 \%$ da matriz energética da região oriunda da lenha obtida por meio da exploração não sustentável, e $70 \%$ das famílias da região utilizam lenha para suas demandas domésticas, bem como fornece uma série de produtos florestais não madeireiros (MMA, 2010).

O manejo intenso e mal planejado desse recurso, em áreas extremamente sensíveis do ponto de vista climático e ambiental, ocasiona alta degradação do solo que se não for controlada pode levar ao processo irreversível de desertificação.

A desertificação é um fenômeno que resulta da combinação de fatores naturais, principalmente as episódicas secas, e de fatores antrópicos, como o sobrepastoreio, desmatamento, atividades agrícolas e mineração, que ocorrem acima da capacidade de suporte do ambiente (LANFREDI et al., 2015; VIEIRA et al., 2015).

Atualmente, há uma preocupação mundial com o aumento das áreas desertificadas, uma vez que os impactos ocasionados pela degradação nos recursos da terra são reconhecidos como um problema ambiental global (LANFREDI et al., 2015). Para essas áreas frágeis, torna-se necessário propor estratégias que permitam o monitoramento contínuo e preciso, visando não somente avaliar a vulnerabilidade, mas também frear o processo de degradação (IBAÑEZ et al., 2014; ZDRULI, 2014).

Para melhor compreender os mecanismos de feedbacks que ocorrem nas regiões semiáridas, é necessário considerar recortes territoriais que permitam analisar possíveis tendências espaçotemporais de variáveis ambientais, que contribuam para a identificação de áreas em que o processo de degradação/desertificação do solo está associado à perda da biodiversidade.

Independente do fator envolvido, a degradação das áreas naturais quase sempre se inicia com o desmatamento e com a substituição da vegetação nativa por outra cultura e/ou ciclo de vida diferente. Segundo Araújo-Filho (2015), em áreas de Caatinga é muito frequente a alteração da vegetação nativa por pastos herbáceos ou culturas de ciclo curto. A retirada da cobertura vegetal torna a região mais susceptível ao processo de desertificação, uma vez que ela protege a paisagem de diversas maneiras, como, por exemplo, evitando o ingresso imediato das águas provenientes das precipitações pluviais nas correntes de drenagem, que provoca incremento do escoamento superficial (runoff), com o consequente aumento na capacidade de erosão (CREPANI, 2001; GUERRA; CUNHA, 2003).

Além disso, a substituição da pastagem natural e da cobertura da vegetação lenhosa em área de solo exposto afeta a temperatura do solo e a umidade do ar e, portanto, influencia os movimentos das massas atmosféricas e das chuvas (FAO, 2000). Embora muitas pesquisas tenham avaliado o papel do clima e dos fatores antrópicos que podem desencadear o processo de desertificação (PRINCE et al., 1998; NICHOLSON, 2005; WANG et al., 2006; XU et al., 2010; WANG et al., 2012; VIEIRA et al., 2015), ainda persistem muitas discordâncias acerca das metodologias para identificação de áreas sob alto processo de degradação do solo (WANG et al., 2006; ZHENG et al., 2006). As relações entre a desertificação, a seca e a influência humana são extremamente complexas.

Existem vários indicadores que podem auxiliar na identificação de áreas desertificadas, entre eles incluem-se elevações da temperatura média e da amplitude térmica diurna, déficit hídrico dos solos, intensificação da erosão eólica, etc. No entanto, o aumento do período seco e a redução da precipitação ao longo de séries temporais superiores a 30 anos são considerados os mais significativos para esse tipo de estudo (CONTI, 1991).

Com base nessas premissas, o presente trabalho baseia-se na análise espaço-temporal das mudanças de usos e cobertura da terra associadas aos padrões de temperatura e de precipitação, com a finalidade de determinar padrões de respostas que auxiliem na identificação de áreas degradadas. Para isso, selecionou-se uma área que apresenta alta severidade à degradação/desertificação do solo, sendo essa localizada no semiárido brasileiro, considerado um dos mais populosos do mundo segundo Marengo (2008) e o mais vulnerável às mudanças climáticas (IPCC, 2007). 


\section{MATERIAIS E MÉTODOS}

\section{1 ÁREA DE ESTUDO}

A região de estudo selecionada, conhecida como núcleo de desertificação de Cabrobó, está situada na faixa próxima à zona equatorial (8-9오, 38-40W), totalizando uma extensão de 12.812,53 Km2 (Figura 1).

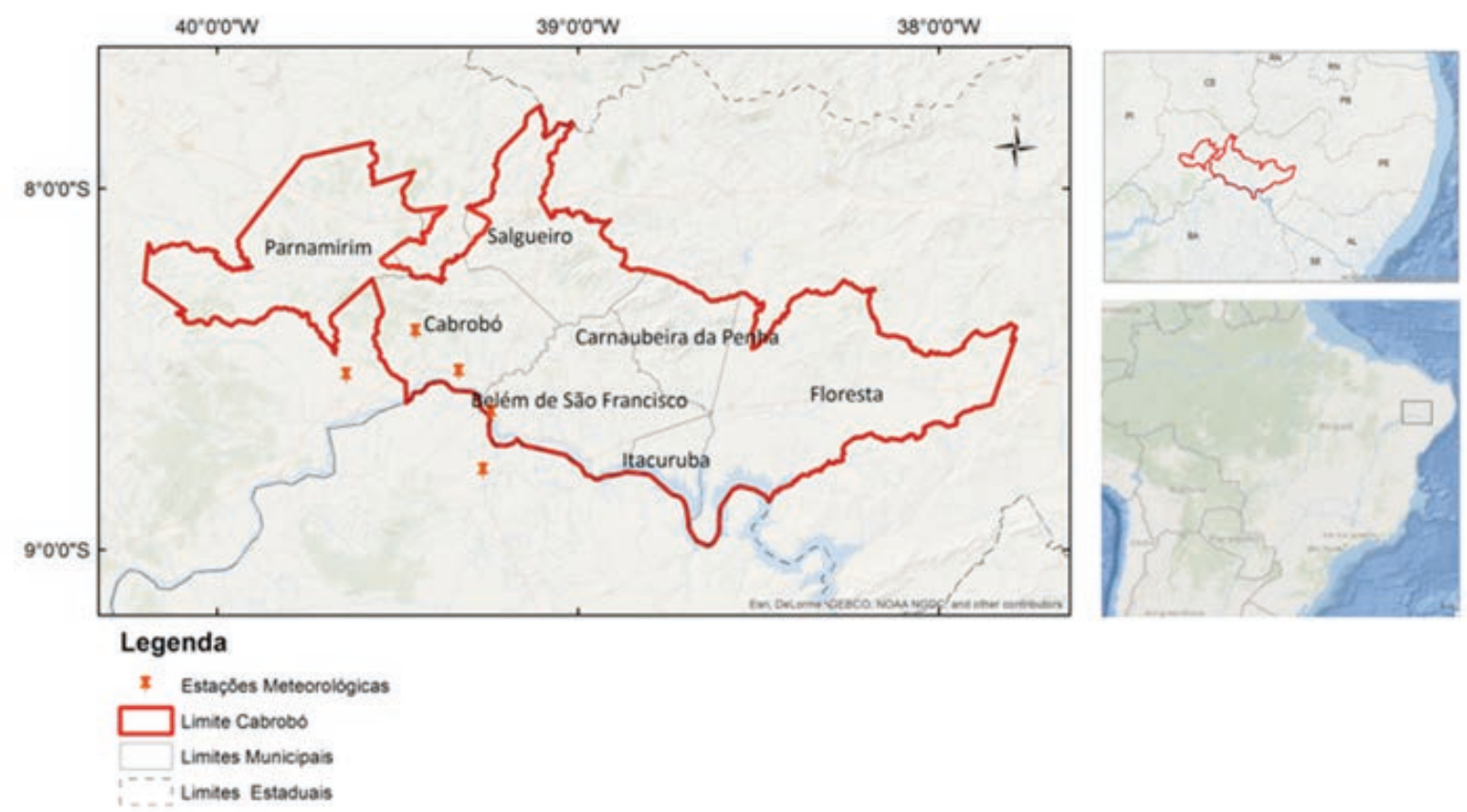

Figura 1 - Localização da área de estudo.

Fonte: Elaborada pelos autores.

Grande parte da região apresenta solos muito rasos e pedregosos, com baixa retenção de água (SÁ et al., 2006). O clima é semiárido, com precipitação média anual de aproximadamente $500 \mathrm{~mm}$. Em termos de médias mensais, para um período de 55 anos, observa-se que a precipitação concentra-se nos meses de janeiro a abril, indicando a existência de uma estação seca intensa e duradoura entre os meses de maio a novembro (Figura 2a). As temperaturas mínimas e máximas mensais situam-se entre $22^{\circ} \mathrm{C}$ e $32^{\circ} \mathrm{C}$ (média anual de $27^{\circ} \mathrm{C}$, Figura $2 \mathrm{~b}$ ), respectivamente. Além disso, a região apresenta características climáticas extremas, tais como elevada incidência de radiação solar, baixas taxas de umidade relativa, elevada evaporação e baixa nebulosidade. Os rios que cortam a região são em sua maioria intermitentes, com exceção do Rio São Francisco, enquanto o tipo de vegetação dominante é a Caatinga (SOARES, 2012). 


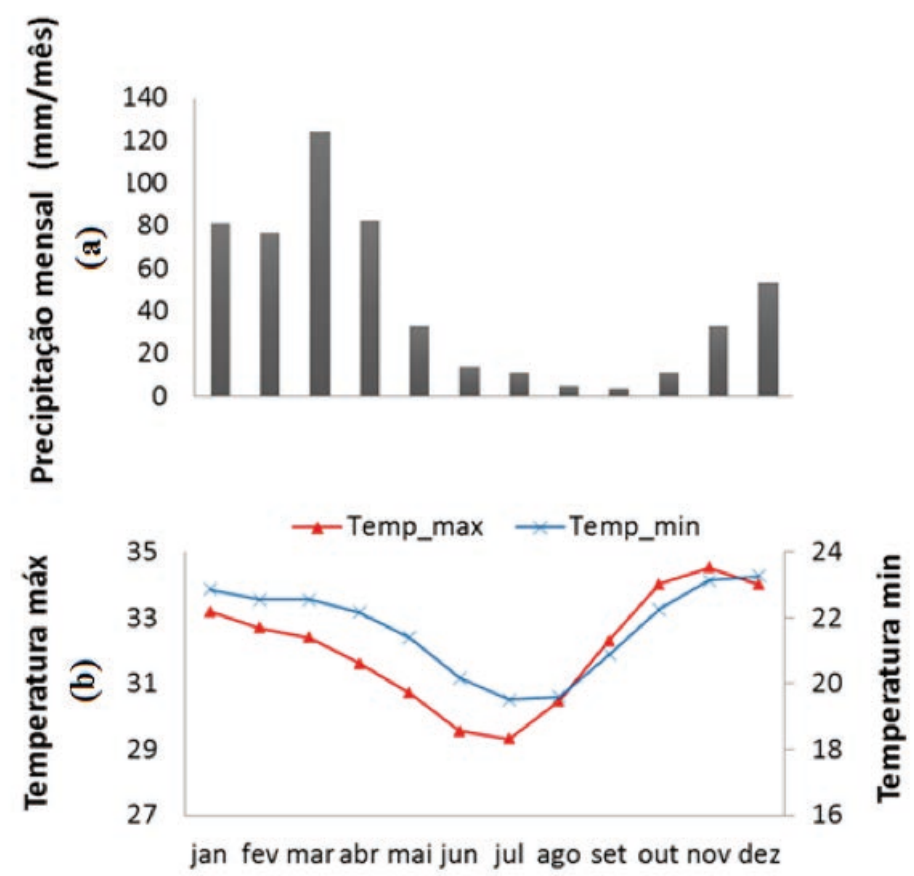

Figura 2 - Precipitação pluviométrica média mensal $(\mathrm{mm})$ e temperaturas máximas e mínimas médias das estações descritas na Tabela 1.

Fonte: Elaborada pelos autores.

\subsection{DADOS IN SITU}

\section{DADOS DE PRECIPITAÇÃO}

Os dados observacionais de precipitação considerados no estudo são provenientes da Rede Hidrometeorológica Nacional da Agência Nacional de Águas (ANA), coletados via ferramenta Hidroweb (SNIRH, 2017). As estações selecionadas estão localizadas próximas ou inseridas dentro do limite do núcleo de desertificação de Cabrobó (Figura 1 e Tabela 1). A partir dos dados de precipitação das estações meteorológicas, elaborou-se uma única série temporal com a finalidade de se obter informações mais completas e com menos falhas para o período de 1961 a 2016 (dados esses referenciados no presente trabalho como PREC-ANA).

Tabela 1 - Estações pluviométricas do banco de dados Hidroweb da ANA.

\begin{tabular}{|c|c|c|c|}
\hline Nome da Estação & Código & Latitude & Longitude \\
\hline Cabrobó & 839003 & $-8,500$ & $-39,316$ \\
\hline $\begin{array}{c}\text { Fazenda Macam- } \\
\text { bira }\end{array}$ & 839007 & $-8,399$ & $-39,449$ \\
\hline Fazenda Tapera & 839034 & $-8,520$ & $-39,641$ \\
\hline Curral das Pedras & 839046 & $-8,783$ & $-39,262$ \\
\hline Ibó & 839031 & $-8,622$ & $-39,239$ \\
\hline
\end{tabular}

Fonte: Elaborada pelos autores. 


\section{ANÁLISES DAS CONDIÇÕES CLIMÁTICAS DA REGIÃO}

Para analisar as condições climáticas da região de estudo, calculou-se a climatologia da precipitação e das temperaturas máxima e mínima, assim como foi calculado o Índice Padronizado de Seca (Standardized Precipitation Index - SPI). A climatologia, tanto da precipitação quanto das temperaturas, foi calculada por meio da equação 1 .

$\operatorname{Clim}_{\mathrm{ij}}=\mathrm{X}_{\mathrm{ij}}-\left(\mathrm{X}_{\mathrm{j}}\right)$

em que $\operatorname{Clim}_{\mathrm{ij}}$ é a climatologia da temperatura ou da precipitação para o mês i e o ano j, $X_{i j}$ é o valor da variável analisada durante o mês i e o ano $\mathrm{j},\left(X_{\mathrm{i}}\right)$ é a média dos valores do mês i em relaçã̃o a todos os anos da série.

A metodologia de cálculo do SPI se resume ao ajuste de uma série de dados acumulados de precipitação na função Gama, sendo sob essa distribuição de probabilidades aplicada uma distribuição inversa Gaussiana (mais detalhes quanto a essa metodologia podem ser consultados em Mckee et al., 1993). Para o presente estudo, calculou-se o SPI com um acumulado móvel de 12 meses de precipitação para o período de 1961 a 2016. O SPI pode ser utilizado para identificar quando a precipitação de uma determinada região se encontra abaixo do estatisticamente esperado e, consequentemente, associar isso à ocorrência de um evento de seca. Assim, neste trabalho considerou-se seca o período em que o SPI é continuamente negativo e atinge um valor igual ou inferior a -1. 0 término de um evento de seca identificado pelo SPI se dá quando este alcança um valor superior a zero (MISHRA et al., 2009; SPINONI et al., 2014, 2015).

\section{DADOS DE PRECIPITAÇÃO INTERPOLADOS}

Uma segunda base de dados de precipitação disponível no Centro de Previsão de Tempo e Estudos Climáticos (CPTEC/INPE), a qual inclui dados oriundos de diversas fontes, tais como do Instituto Nacional de Meteorologia (INMET) e de Centros Estaduais de Meteorologia, foi utilizada para a avaliação espacial da precipitação na área de estudo (aqui denominada PREC-CPTEC). Essa base consiste de dados observados de estações meteorológicas e interpolados em grade regular de $5 \mathrm{~km}$ de resolução espacial utilizando a técnica de kriging (MATHERON, 1969). O período de dados considerado foi de 2000 a 2014.

\subsection{DADOS ORBITAIS}

\section{TEMPERATURA DA SUPERFÍCIE}

Os dados utilizados para compor a variável temperatura de superfície $-T_{s}$ são provenientes do sensor Moderate Resolution Imaging Spectroradiometer (Modis), produto MOD11A2 (Land Surface Temperature - LST), coleção 5, com resolução espacial de $1 \mathrm{~km}$. A informação sobre temperatura de superfície gerada por esse sensor utiliza o algoritmo LST para o cálculo de TS, incluindo o Day/Night LST algorithm (WAN; LI, 1997). Esse algoritmo foi desenvolvido especificamente para o sensor Modis e produz imagens termais diurnas e noturnas para toda a superfície da Terra, com periodicidade diária.

O produto A2 é uma composição de oito dias, obtida a partir de dados diários gerados pelo produto A1. Entre as duas plataformas disponíveis para a obtenção dos dados de temperatura de superfície (Terra e Água), optou se pela utilização do satélite Terra, visto que análises preliminares mostraram que as informações obtidas por meio dessa plataforma apresentam menor contaminação das informações por nuvens, após a avaliação das imagens de Quality Control - QC, que acompanha cada imagem LST. Como os dados originais do produto MOD11 são fornecidos em graus Kelvin, foi necessário utilizar um fator de conversão para que os dados da imagem fossem transformados para graus Celsius, de acordo com a equação 2 : 
$\mathrm{TSC}=(\mathrm{B} 1 * 0,02)-273$

em que TSC é o valor do pixel em graus Celsius, B1 é o valor original do pixel em graus Kelvin e 0,02 é o fator de escala.

\section{NORMALIZED DIFFERENCE VEGETATION INDEX - NDVI}

Para a classificação do solo exposto, foram utilizadas imagens do sensor Modis com resolução espacial de 250 m, obtidas do subproduto MOD13Q referente ao Índice de Vegetação da Diferença Normalizada (Normalized Difference Vegetation Index - NDVI). O NDVI é calculado subtraindo-se a banda espectral do infravermelho próximo pela banda do vermelho e dividindo-se esse resultado pela soma das duas. Valores positivos desse índice são associados às condições da vegetação, uma vez que quando as plantas estão mais saudáveis ocorre uma maior reflectância da banda do infravermelho próximo e menor na banda do vermelho. As imagens do produto MOD13Q são disponibilizadas em mosaicos de 16 dias, já com correção atmosférica, livres de nuvens e georreferenciadas.

A etapa inicial do trabalho compreendeu a aquisição de todos os arquivos disponíveis para a área e período de estudo (2000 a 2016). Em seguida, obtiveram-se as médias dos períodos seco e úmido para cada ano, levando em consideração a delimitação das estações definidas por Cunha et al., 2015. A análise inicial do conjunto de dados da série multitemporal dos valores médios de NDVI para as estações seca e úmida foi realizada com o propósito de identificar os pixels com padrão de solo exposto. Logo, para o pixel ser considerado como solo exposto foi aplicada a seguinte regra para cada ano do período de análise:

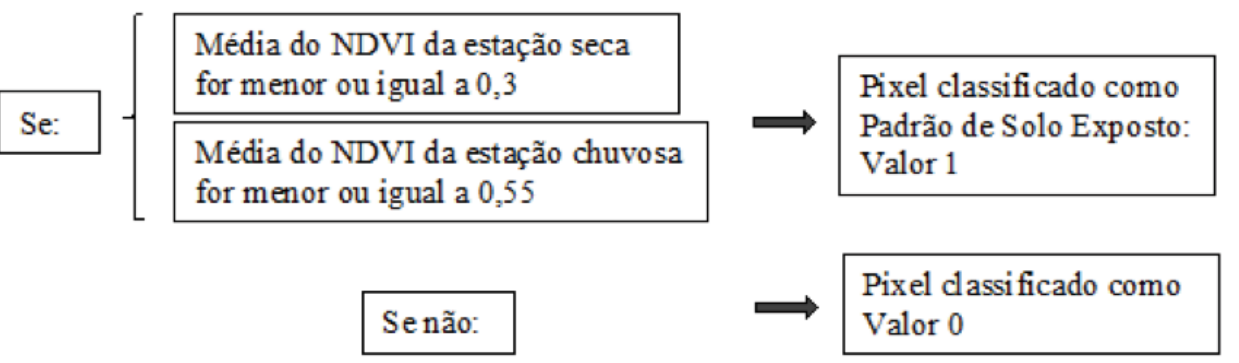

Figura 3 - Método de seleção dos pixels de solo exposto.

Fonte: Elaborada pelos autores.

Os limiares de NDVI foram selecionados com base em trabalho de campo realizado no Alto Sertão Sergipano, uma vez que essa região apresenta características ambientais semelhantes à de Cabrobó, sendo também uma área com severo processo de degradação.

\section{DADOS REFERENTES ÀS ÁREAS DEGRADADAS}

As áreas degradadas foram obtidas do Projeto de Sistema de Alerta Precoce contra Seca e Desertificação - SAP desenvolvido pelo Instituto Nacional de Pesquisas Espaciais - INPE e o Ministério do Meio Ambiente - MMA. Essas áreas foram identificadas segundo índice de degradação baseado em dados de NDVI, que considera a frequência e persistência do solo exposto em certo período de tempo. Para isso, foram atribuídos pesos para a degradação do solo que variaram linearmente entre o primeiro e o último ano analisado. $O$ valor do peso varia entre 0 , que indica que o solo exposto não permaneceu por todos os anos analisados e 1, que indica que os dados de solo exposto têm exatamente o mesmo peso e permaneceram por toda a série analisada (TOMASELLA et al., 2018). 


\subsection{DADOS DE USO E COBERTURA DA TERRA}

O mapa de uso e cobertura da terra foi elaborado com base nas informações fornecidas pelo projeto MapBiomas referente à coleção 2.3, que corresponde ao período de 2000 a 2016 (MAPBIOMAS, 2017).

Para a utilização no presente trabalho, após o download dos mapas, as classes foram agregadas visando facilitar as análises de mudanças de usos e cobertura da terra, conforme ilustrado na Tabela 2.

Tabela 2 - Classes agregadas do MapBiomas

\begin{tabular}{|c|c|}
\hline Classes MapBiomas & Classe atual \\
\hline $\begin{array}{l}\text { Floresta Densa } \\
\text { Floresta Aberta } \\
\text { Floresta Secundária } \\
\text { Floresta Alagada } \\
\quad \text { Mangue } \\
\text { Floresta Degradada }\end{array}$ & Floresta \\
\hline $\begin{array}{c}\text { Vegetação Campestre } \\
\text { Pastagem } \\
\text { Outras Pastagens } \\
\text { Pastagem em Campos Naturais } \\
\text { Uso Agropecuário }\end{array}$ & Pastagem \\
\hline $\begin{array}{c}\text { Culturas Anuais } \\
\text { Culturas Semiperene (Cana-de-Açúcar) } \\
\text { Sivicultura }\end{array}$ & Agricultura \\
\hline $\begin{array}{c}\text { Formações Naturais não Florestais } \\
\text { Formações Úmidas Naturais não Florestais } \\
\text { Outras Formações não Florestais } \\
\text { Corpos-d'Água } \\
\text { Infraestrutura Urbana }\end{array}$ & Outros \\
\hline
\end{tabular}

Fonte: Elaborada pelos autores.

\section{RESULTADOS E DISCUSSÕES}

\subsection{CONDIÇÕES CLIMÁTICAS DA REGIÃO}

A Figura 4 apresenta o SPI calculado para a região de estudo, sendo os períodos de secas representados em vermelho e os períodos mais chuvosos ilustrados na cor azul.

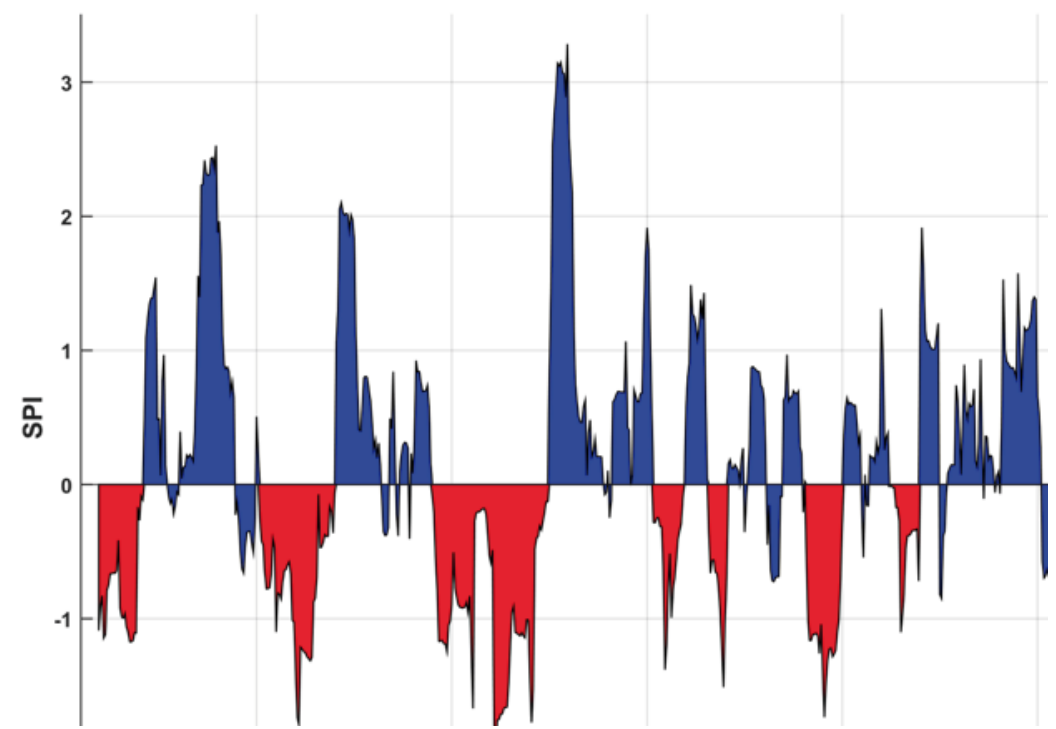

Figura 4 - SPI calculado com acumulado móvel de 12 meses para a região de estudo.

Fonte: Elaborada pelos autores. 
Analisando a Figura 4 pode-se constatar que a região do Núcleo de Cabrobó é afetada frequentemente por eventos de seca, e que após a segunda metade do período analisado houve um aumento na frequência da ocorrência desse tipo de fenômeno. Outra característica que pode ser observada é que o último evento de seca foi o mais intenso (SPI mais negativo) e o segundo mais duradouro dentro da série analisada, condição esta que pode ter contribuído para o aumento da variação da área degradada e de solo exposto em relação aos demais anos.

Segundo Brito et al. (2018), a última grande seca na região Nordeste teve início no final de 2011, se intensificou em toda a região no ano de 2012 e permaneceu até o ano de 2017, sendo considerado o pior evento de seca das últimas décadas. A Figura 5 apresenta os percentuais de anomalias de precipitação identificadas a partir da análise dos dados coletados do CPTEC-INPE. Nota-se que o ano de 2011 foi o que apresentou os menores percentuais de anomalias de chuva em toda a área de estudo, condição essa que se manteve no ano de 2015.
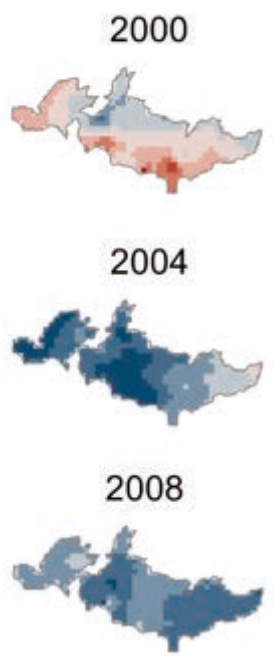

2012
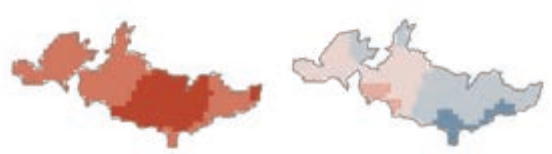

$=<-80$
$=-80-60$
$=-60--80$
$=-40--20$
$=-20-0$

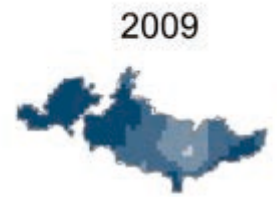

2013
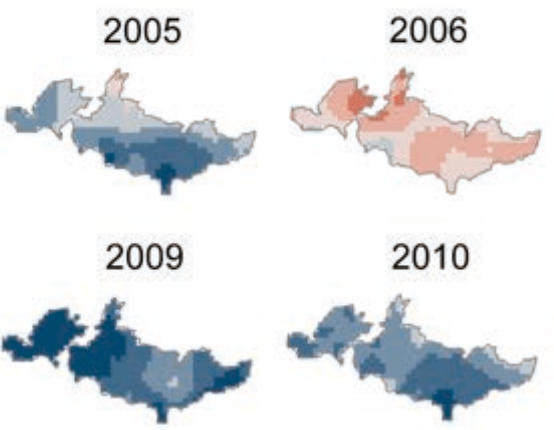

2014
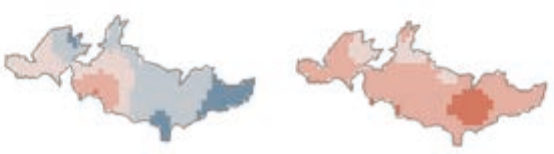

Figura 5 - Percentual de anomalias de precipitação (\%) (PREC-CPTEC).

Fonte: Elaborada pelos autores.

Os efeitos da seca se acumulam lentamente ao longo de um período considerável de tempo e podem perdurar anos após a finalização do evento, conforme ressaltado por Batista Júnior (2012). Um exemplo é a última seca observada na região semiárida (2011-2017), que depois do sexto ano consecutivo de déficit de precipitação, mesmo com registros de acumulados de chuva acima da média climatológica (início de 2018), principalmente na porção norte da região, grande parte dos reservatórios permanece com volumes muito baixos (críticos), podendo levar meses para que a situação se aproxime das condições normais. Assim, a recorrência de seca pode causar modificações na umidade do solo e na recarga dos aquíferos, interferindo na disponibilidade de águas subterrâneas e no escoamento superficial (IPCC, 2007(a); IPCC, 2013). 
As projeções futuras de clima indicam riscos de secas intensas no semiárido, reduções de chuva em até $40 \%$ e aumento de dias secos consecutivos. Além disso, os mais frequentes e intensos anos de El Niño (Enso) causados pelas mudanças climáticas podem também aumentar a escassez hídrica e o risco de secas (ALVES; REPELLI, 1992; MARENGO, 2008). Esses aspectos, associados ao crescimento populacional e à demanda crescente por água, podem levar à insegurança alimentar e aumentar a vulnerabilidade dos agricultores mais pobres (PBMC, 2012), afetando a economia da região, uma vez que inviabiliza a implementação de agriculturas de sequeiros, conforme salientado por Ribeiro (2003). Os impactos dessas alterações poderão afetar diretamente cerca de 7 milhões de pessoas vinculadas à agricultura familiar no nordeste brasileiro, e que frequentemente se encontram em condições de extrema pobreza (IBGE, 2006; LINDOSO et al., 2011).

Como alternativa à recorrência de episódios de déficit hídrico na região, torna-se necessária a utilização de métodos de irrigação adequados, pois estes, quando realizados de forma inadequada, acarretam a salinização do solo, levando ao abandono das terras pelos agricultores (OLIVEIRA SILVA; BARROS SILVA, 2015).

As secas também afetam a capacidade de resiliência do ambiente, ocasionando impacto negativo na dinâmica dos processos pedogenéticos e acentuando as ações morfodinâmicas, causando desequilíbrios ecológicos (CGEE, 2016).

\subsection{AVALIAÇÃO DAS MUDANÇAS DE USO E COBERTURA DA TERRA}

De modo geral, pode-se constatar, analisando-se a Figura 6, que a cobertura natural da região vem sendo substituída por áreas de solo exposto, aumentadas em 349\% entre os anos de 2000 (1503 km2) e 2010 (6753 km2). A partir de 2012, quando se inicia o período mais intenso de seca, as alterações são mais evidentes, verificando-se a substituição de áreas de floresta e de pastagens por solo exposto, sendo constatada uma perda de cobertura vegetal de $37 \%$.

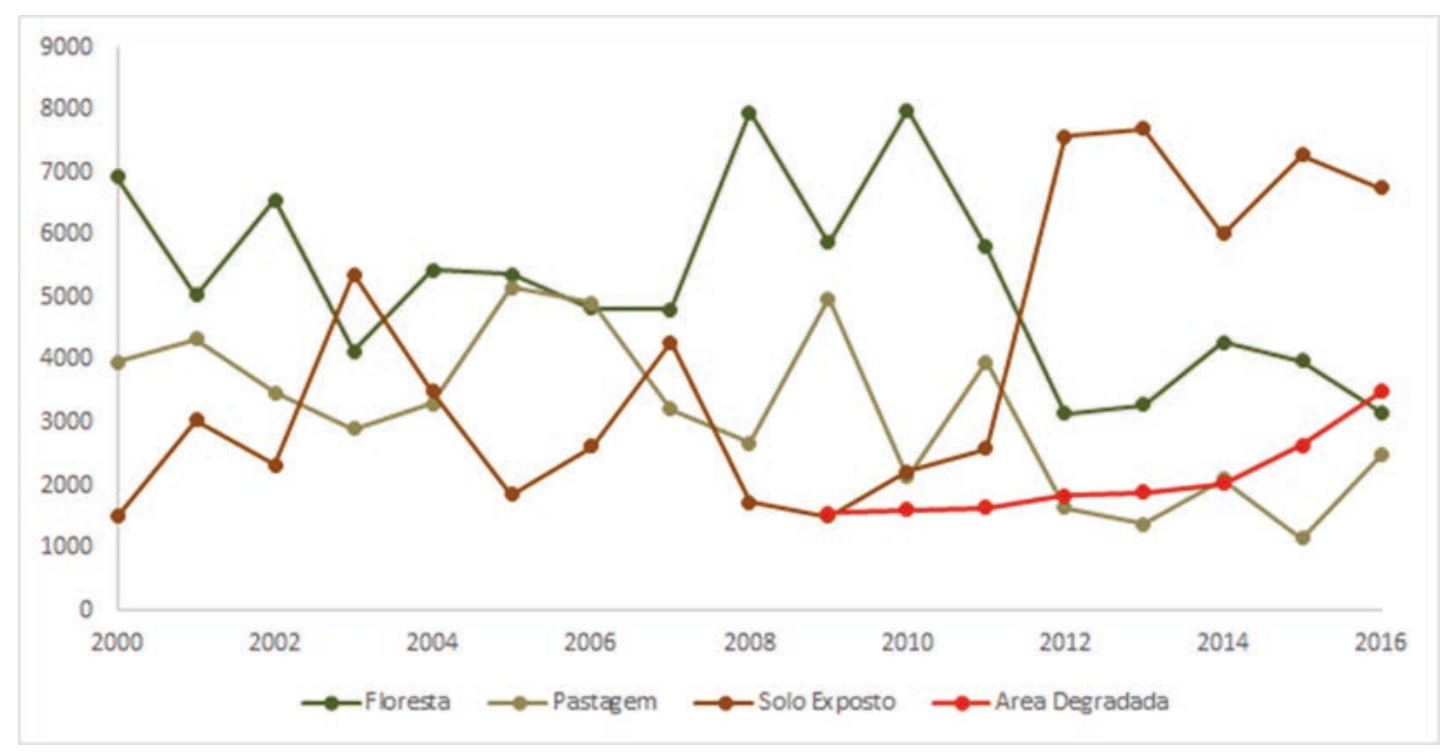

Figura 6 - Mudanças de uso e cobertura da terra (km2).

Fonte: Elaborada pelos autores.

As áreas degradadas que, pela metodologia do SAP passam a ser contabilizadas a partir de 2009, também estão se expandindo, tendo sido identificado um aumento de $125 \%$ entre o período analisado. Essas áreas concentram-se na região centro-sul da área de estudo (Figura 7), embora a partir de 2012 seja possível observar que elas estão se expandindo para o oeste. 
Segundo Sampaio et al. (2003), grande parte dessa região apresenta problemas de aridez e solos rasos, forçando a população a se localizar de forma mais concentrada em ilhas do Rio São Francisco e nos baixios mais férteis, com consequente aumento da pressão antrópica nessas regiões. Segundo os autores, o principal problema da região está relacionado à salinização dos solos em função do plantio de alho e cebola com irrigação e condições de drenagem inadequadas. Além da questão da salinidade, a região também apresenta pecuária extensiva, o que tem levado ao sobrepastoreio (SALES, 2006).

O aumento das áreas mapeadas como degradadas, no presente trabalho, é preocupante em relação à desertificação, uma vez que nessas regiões não se observa o crescimento de nenhum tipo de cobertura vegetal ou agricultura por longos períodos de tempo (pelo menos 17 anos), indicando serem áreas em acelerado processo de degradação do solo.

As alterações de áreas de vegetação nativa em áreas degradadas, de solo exposto e com pastagens levam ao aumento do albedo da superfície, que afeta o balanço de radiação da superfície e controla a quantidade de energia disponível para o aquecimento e a evaporação de água na baixa atmosfera.

Cunha et al. (2013) observaram que em áreas onde houve a conversão de caatinga natural para caatinga degradada e agropecuária, o aumento do albedo médio anual da superfície foi de 0,12 e 0,16, respectivamente, sendo ainda maior durante a estação de seca, devido à redução de folhas verdes em função do baixo índice pluviométrico e baixa umidade do solo. Todas essas características associadas às altas temperaturas podem acelerar o processo de desertificação (SOARES, 2012).

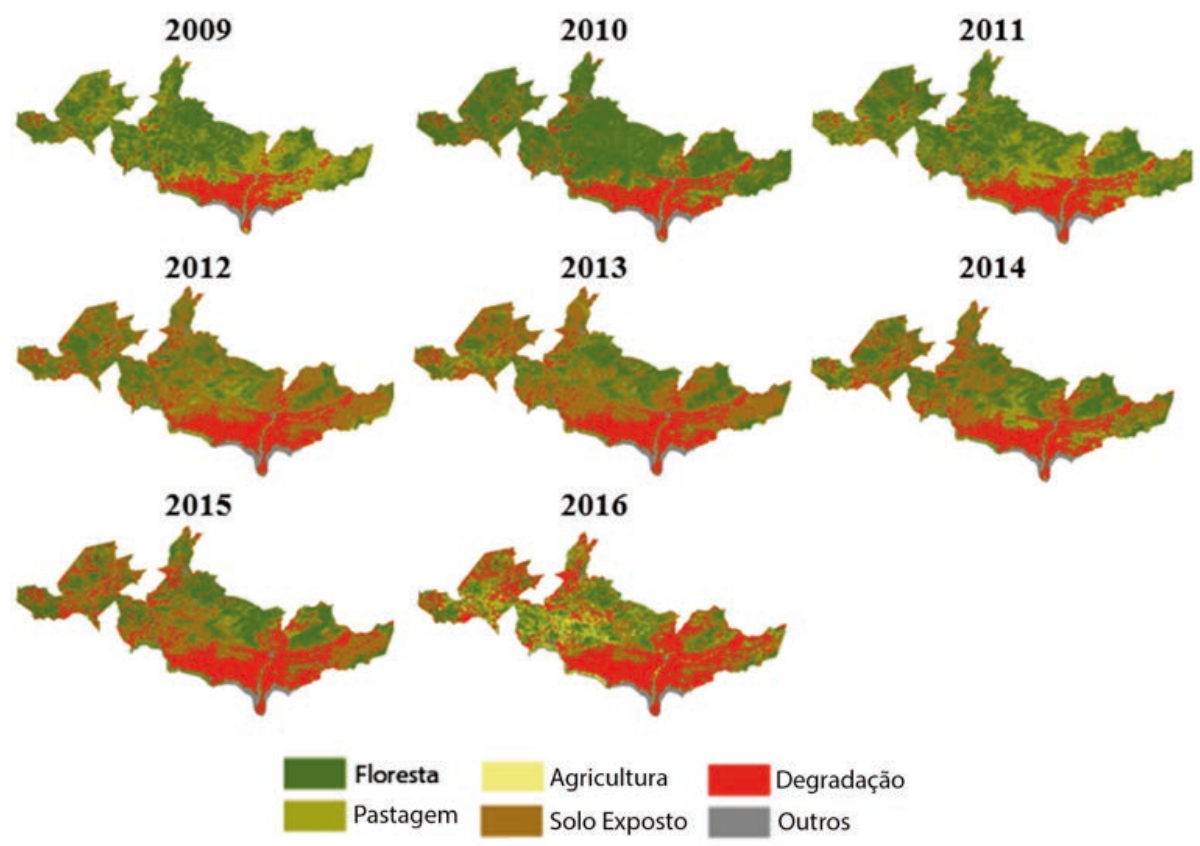

Figura 7 - Mapeamento de uso e cobertura da terra.

Fonte: Elaborada pelos autores.

Outro fator que pode estar contribuindo para acelerar o processo de degradação da terra é a transposição do Rio São Francisco, que intensifica o desmatamento às margens do rio devido à execução de obras de terraplanagens (MI, 2017). A região de estudo é o ponto de partida para a transposição, saindo dos municípios de Cabrobó e Floresta diversos canais que são divididos em dois eixos, um na direção norte e outro na direção leste, que percorrem toda a área de caatinga levando à fragmentação da paisagem (GUIMARÃES JÚNIOR, 2010). O governo Pernambucano autorizou a supressão de 516 mil hectares de vegetação nativa para a realização das obras. Em Cabrobó estava previsto um desmatamento de 83,38 mil hectares; no entanto, em 2012, o valor triplicou para 1.576 hectares (ALEPE, 2012). Pelos cálculos de área apresentados na Tabela 3, é possível constatar que entre os anos de 2000 a 2016 a região já perdeu $54 \%$ de vegetação nativa. 
Tabela 3 - Cálculo de área $(\mathrm{km} 2)$ das classes de uso e cobertura da terra.

\begin{tabular}{l|c|c|c|c|c}
\hline Ano & \multicolumn{1}{l}{ Floresta } & \multicolumn{1}{c}{ Pastagem } & \multicolumn{1}{c}{ Agricultura } & \multicolumn{1}{c}{ Solo Exposto } & Área Degradada \\
\hline $\mathbf{2 0 0 0}$ & 6923,30 & 3969,74 & 0,08 & 1503,01 & \\
\hline $\mathbf{2 0 0 1}$ & 5032,44 & 4333,39 & 0,03 & 3043,99 & \\
\hline $\mathbf{2 0 0 2}$ & 6568,56 & 3477,78 & 0,07 & 2328,05 & \\
\hline $\mathbf{2 0 0 3}$ & 4138,72 & 2906,03 & 0,11 & 5369,80 & \\
\hline $\mathbf{2 0 0 4}$ & 5446,41 & 3311,97 & 0,17 & 3502,36 & \\
\hline $\mathbf{2 0 0 5}$ & 5371,53 & 5153,08 & 0,08 & 1856,46 & \\
\hline $\mathbf{2 0 0 6}$ & 4826,42 & 4917,77 & 0,34 & 2629,29 & \\
\hline $\mathbf{2 0 0 7}$ & 4814,72 & 3212,34 & 0,05 & 4290,09 & \\
\hline $\mathbf{2 0 0 8}$ & $\mathbf{7 9 5 6 , 1 2}$ & 2672,36 & 0,07 & 1735,90 & \\
\hline $\mathbf{2 0 0 9}$ & 5881,88 & 4986,73 & 0,09 & 1499,35 & 1559,54 \\
\hline $\mathbf{2 0 1 0}$ & 8001,21 & 2132,47 & 0,07 & 2211,51 & 1613,25 \\
\hline $\mathbf{2 0 1 1}$ & 5808,00 & 3978,21 & 0,24 & 2578,54 & 1639,47 \\
\hline $\mathbf{2 0 1 2}$ & 3150,70 & 1642,05 & 0,05 & 7582,43 & 1827,50 \\
\hline $\mathbf{2 0 1 3}$ & 3288,36 & 1372,89 & 0,20 & 7708,75 & 1884,95 \\
\hline $\mathbf{2 0 1 4}$ & 4292,51 & 2091,61 & 0,05 & 6019,21 & 2023,09 \\
\hline $\mathbf{2 0 1 5}$ & 3983,11 & 1158,45 & 0,11 & 7281,91 & 2636,00 \\
\hline $\mathbf{2 0 1 6}$ & 3160,69 & 2484,78 & 0,12 & 6752,92 & 3503,24 \\
\hline
\end{tabular}

Fonte: Elaborada pelos autores.

Com relação à tendência de temperatura da superfície para os diferentes tipos de usos e cobertura da terra é possível observar que esta é positiva a partir do ano de 2010 para todas as classes (Figura 8). No entanto, os valores são mais elevados sobre a área degradada $\left(38^{\circ} \mathrm{C}\right)$, seguida da área de solo exposto $\left(37^{\circ} \mathrm{C}\right)$ e de pastagem $\left(36^{\circ} \mathrm{C}\right)$. As temperaturas mais baixas foram registradas em 2008 , sobre áreas de Floresta $\left(32^{\circ} \mathrm{C}\right)$. Esses resultados corroboram os encontrados por Souza et al. (2016) que, ao investigar os impactos das mudanças de uso da terra na temperatura, identificaram valores inferiores de temperatura da superfície para as classes água e vegetação arbórea/arbustiva e valores mais elevados sobre as classes de solo exposto, áreas urbanas e vegetação herbácea respectivamente. Segundo Chagnon (1992) e Foley et al. (2005), em áreas desmatadas a temperatura tende a aumentar devido à mudança na distribuição de energia. Em áreas com menor disponibilidade de umidade, a partição de energia se dá de forma que a maior parte é liberada em forma de calor sensível, enquanto o calor latente diminui. Já em áreas com cobertura vegetal, a evaporação converte a energia solar em água evaporada em vez de calor (MASHIKI; CAMPOS, 2013; CUNHA et al., 2013).

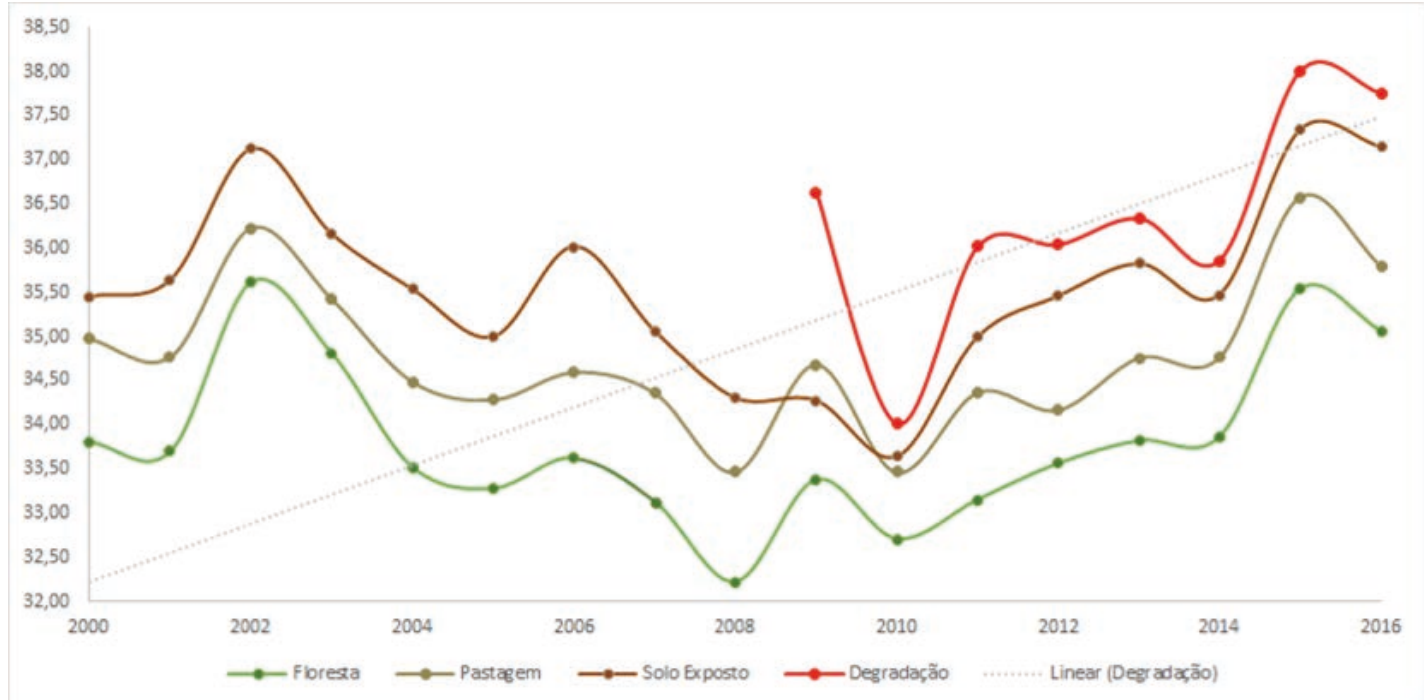

Figura 8 - Relação entre os valores médios de temperatura $\left({ }^{\circ} \mathrm{C}\right)$, extraídos do Modis, e as classes de uso e cobertura da terra. 


\section{CONCLUSÕES}

A partir de informações oriundas de sensoriamento remoto juntamente com técnicas de geoprocessamento, foi possível analisar as relações existentes entre os dados de clima (precipitação e temperatura) e as mudanças de uso e cobertura da terra na região do núcleo de desertificação de Cabrobó, em Pernambuco.

O produto MOD11A2, proveniente do sensor Modis, mostrou-se adequado para o monitoramento da temperatura da superfície. Em razão da alta frequência temporal e espacial dos dados, é possível acompanhar alterações de temperatura e como estas se comportam diante das mudanças de uso e cobertura da terra. Além disso, a utilização de dados provenientes de sensoriamento remoto supre a carência de informações in situ, principalmente pela falta de estações meteorológicas densamente distribuídas.

As áreas degradadas foram as que apresentaram valores mais elevados de temperatura da superfície; logo, é possível assumir que nas regiões onde há maior risco de degradação/desertificação há um agravamento do déficit hídrico dos solos. $\mathrm{O}$ aumento da temperatura da superfície sobre as áreas degradadas foi de $1^{\circ} \mathrm{C}$. Por outro lado, a partir dos dados de precipitação não foi possível identificar padrões de anomalias que pudessem estar associados às áreas degradadas ou de solo exposto, devido ao curto período avaliado.

Finalmente, como contribuição do presente trabalho, destaca-se a possibilidade de acompanhar as mudanças de uso e cobertura da terra por um longo período de tempo (17 anos), bem como a viabilidade de associar essas mudanças com dados de clima (em especial de precipitação e de temperatura da superfície). Além disso, a utilização de dados de NDVI para a identificação de áreas de solo exposto e a inclusão do índice de áreas degradadas na legenda do mapa de uso e cobertura da terra possibilitaram identificar padrões de temperatura da superfície que podem ser utilizados para auxiliar no monitoramento de áreas com maior risco de degradação/desertificação.

A espacialização dessas informações permite diagnósticos mais precisos das localidades onde estão ocorrendo mudanças na paisagem, o que possibilita agilizar tomadas de decisão e subsidiar a distribuição de recursos financeiros para recuperação dessas áreas. Considerando ainda que a população em áreas rurais depende da agricultura de subsistência e de sequeiro, os resultados deste estudo são relevantes para futura avaliação do risco de seca, a qual é definida como a ameaça física (eventos de seca - déficit de chuva) associada às vulnerabilidades locais, que inclui, além das questões socioeconômicas, aquelas relacionadas ao ambiente (p.e. regiões susceptíveis à degradação).

\section{REFERÊNCIAS}

ALEPE (Assembleia Legislativa de Pernambuco). Lei № 14.685, de 31 de Maio de 2012. Disponível em:

<http://legis.alepe.pe.gov.br/arquivoTexto.aspx?tiponorma=1\&numero=14685\&complemento=0\& ano=2012\&tipo=\&url=L0137532009>. Acesso em: set. 2017.

ALVES, J. M. B.; REPELLI, C. A. A variabilidade pluviométrica no setor norte do Nordeste e os eventos El NiñoOscilação Sul (ENOS). Revista Brasileira de Meteorologia, v. 7, n. 2, p. 583-592, 1992.

ARAÚJO FILHO, J. Manejo pastoril sustentável da caatinga (N. IICA L01-52). IICA. Projeto Dom Helder Câmara, Recife (Brasil). Projeto Semear, Brasília (Brasil). Associação Brasileira de Agroecologia, Rio Grande do Sul (Brasil) 2013.

BATISTA JÚNIOR, W. Identificação e avaliação dos fatores de ocorrência de secas na bacia do Rio Guandu Espírito Santo. Tese de Doutorado. Meteorologia Agrícola. Universidade Federal de Viçosa. 2012.

BRASIL. Ministério da Integração Nacional. Projeto São Francisco. Disponível em: <http://www.integracao.gov. br/projeto-sao-francisco1>. Acesso em: 15 set. 2017. 
BRASIL. Ministério do Meio Ambiente. Síntese das discussões e recomendações da componente biodiversidade da caatinga. In: Conferência Internacional: clima, sustentabilidade e desenvolvimento em regiões semiáridas ICID + 18 - 2010, Fortaleza - Ceará, 2010.

BRITO, S. S. B. et al. Frequency, duration and severity of drought in the Semiarid Northeast Brazil region. International Journal of Climatology, v. 38, n. 2, p. 517-529, 2018.

CENTRO DE GESTÃO E ESTUDOS ESTRATÉGICOS. Desertificação, degradação da terra e secas no Brasil, BrasíliaDF, 252 p., 2016.

CHAGNON, S. A. Inadvertent weather modification in urban areas: lessons for global climate change. Bulletin of American Meteorology Society, v. 73, p. 619-627, 1992.

CONTI, J. B. Desertificação em áreas tropicais. III Encuentro de Geografos da America Latina. Toluca, México. Universidad Autonoma del Estado de Mexico. Anais..., v. 2, p. 365-76, 1991.

CREPANI, E. et al. Sensoriamento remoto e geoprocessamento aplicados ao zoneamento ecológico-econômico e ao ordenamento territorial. São José dos Campos: INPE. 2001: 103 (INPE-8454-RPQ/722).

CUNHA, A. P. M. A. et al. Monitoring vegetative drought dynamics in the Brazilian Semiarid Region. Agric. For. Meteorol., v. 214, p. 494-505, 2015.

CUNHA, A. P. M. A.; SAMPAIO, G.; ALVALÁ, R. C. S. Impactos das mudanças de cobertura da superfície nos balanços de energia, água e carbono em uma região semiárida do Brasil. Revista Brasileira de Meteorologia, v. 28, n. 3, 2013.

DE OLIVEIRA SILVA, A. K.; DE BARROS SILVA, H. P. O processo de desertificação e seus impactos sobre os recursos naturais e sociais no município de Cabrobó - Pernambuco -Brasil. PRACS: Revista Eletrônica de Humanidades do Curso de Ciências Sociais da Unifap, v. 8, n. 1, p. 203-215, 2015.

FAO Agriculture: towards 2015/30. Rome: Food and Agriculture Organization, 2001. Technical Interim Report April 2000. Disponível em: <http://www.fao.org/es/ESD/at2015/chapter1.pdf [Geo-2-167]>.

FOLEY J. A. et al. Global consequences of land use. Science, v. 309, p. 570-574, 2005.

GUERRA, A. J. T.; CUNHA, S. B. Geomorfologia: uma atualização de bases e conceitos. Rio de Janeiro: Bertrand Brasil. 2003: 472

GUIMARÃES JÚNIOR, J. A. O destino dos canais da transposição do Rio São Francisco. Revista Cidadania e Meio Ambiente, 2010. Disponível em: <http://www.ecodebate.com.br/2010/04/06/o-destino-dos-canais-datransposicao-do-rio-sao-francisco-artigo-de-joao-abner-guimaraes-jr/>.

IBAÑEZ, J. et al. Multidisciplinary model for assessing degradation in Mediterranean rangelands. Land. Degrad. Dev, v. 25, p. 468-482, 2014.

INSTITUTO BRASILEIRO DE GEOGRAFIA E ESTATÍSTICA. Censo demográfico, Rio de Janeiro, 2010. Disponível em: <https://censo2010.ibge.gov.br/>.

INSTITUTO BRASILEIRO DE GEOGRAFIA E ESTATÍSTICA. Censo Agropecuário, Rio de Janeiro, 2006. Disponível em: $<$ https://www.ibge.gov.br>.

INTERGOVERNMENTAL PANEL ON CLIMATE CHANGE. Climate Change 2013: the physical science basis. Contribution of Working Group I to the Fifth Assessment Report of the Intergovernmental Panel on Climate Change. United Kingdom and New York, p.1535, 2013.

INTERGOVERNMENTAL PANEL ON CLIMATE CHANGE. Climate Change 2007: the physical science basis. Summary for Policy Makers. IPCC Geneva, 2007.

INTERGOVERNMENTAL PANEL ON CLIMATE CHANGE. Climate Change 2007: the physical science basis. Cambridge, p.18, 2007a. 
LANFREDI, M. et al. Early identification of land degradation hotspots in complex Bio-Geographic Regions. Remote Sensing, v. 7, n. 6, p. 8154-8179, 2015.

LINDOSO, D. et al. Climate Change and Vulnerability to drought in the Semiarid: the case of smallholder farmers in the Brazilian northeast. In: MOTTA, R. S. da. (Ed.). Climate change in Brazil: economic, social and regulatory aspects. Brasília: Ipea, p. 235-256, 2011.

MACARTHUR, R. H.; MACARTHUR, J. W. On bird species diversity. Ecology, v. 42, p. 594-598, 1961.

MAPBIOMAS - Coleção 2.3 da Série Anual de Mapas de Cobertura e Uso de Solo do Brasil. Disponível em: <http://mapbiomas.org/>. Acesso em: 07 ago. 2017.

MARENGO, J. A. Vulnerabilidade, impactos e adaptação à mudança do clima no semiárido do Brasil. Parcerias Estratégicas, v. 27, p. 149-75, 2008.

MASHIKI, M. Y.; CAMPOS, S. Influência do uso e ocupação do solo na temperatura aparente da superfície no município de Botucatu-SP. Energia na agricultura, v. 28, n. 30, p. 143-149, 2013.

MATHERON, G. Le krigeage universel. Cahiers du Centre de Morphologie Mathematique, Ecole des Mines de Paris, Fontainebleau, v. 1, 1969.

MCKEE, T. B.; DOESKEN, N. J.; KLEIST, J. The relationship of drought frequency and duration to time scales. In Proceedings of the 8th Conference on Applied Climatology, Boston, MA: American Meteorological Society, v. 17, n. 22, p. 179-183, 1993.

MISHRA, A. K.; SINGH, V. P.; DESAI, V. R. Drought characterization: a probabilistic approach. Stoch. Environ. Res. Risk Assess, v. 23, n. 41, 2009.

NICHOLSON, S. On the question of the "recovery" of the rains in the West African Sahel. Journal of Arid Environments, v. 63, n. 3, p. 615-641, 2005.

PBMC. Sumário Executivo do Volume 1 - Base Científica das Mudanças Climáticas. Contribuição do Grupo de Trabalho 1 para o 1o Relatório de Avaliação Nacional do Painel Brasileiro de Mudanças Climáticas. Brasil, Volume Especial para a Rio+20, 34p, 2012.

PRINCE S. D.; DE COLSTOUN, E. B.; KRAVITZ, L. L. Evidence from rain-use efficiencies does not indicate extensive Sahelian desertification. Global Change Biology, v. 4, n. 4, p. 359-374, 1998.

RIBEIRO, M. R. A pedologia e o planejamento da irrigação no Nordeste do Brasil: uma avaliação crítica. Boletim Informativo da Sociedade Brasileira de Ciência do Solo, v. 28, n. 3, p. 13-15, 2003.

SÁ, I. B.; SÁ, I. D. S.; SILVA, A. D. S. Desertificação na região de Cabrobó-PE: a realidade vista do espaço. In: Embrapa Semiárido - Artigo em anais de congresso (ALICE). In: Simpósio Regional de Geoprocessamento e Sensoriamento Remoto, 3. Aracaju. Anais... Aracaju: Embrapa Tabuleiros Costeiros. 2006.

SALES, M. C. L. O panorama da desertificação no Brasil. In: MOREIRA, E. (Org.). Agricultura familiar e desertificação. João Pessoa: Editora Universitária da UFPB, 2006.

SAMPAIO, E. V. S. B. et al. Desertificação no Brasil: conceitos, núcleos e tecnologias de recuperação e convivência. Recife: Editora Universitária da UFPE, 2003.

SISTEMA NACIONAL DE INFORMAÇÕES SOBRE RECURSOS HÍDRICOS. Acervo de dados hidrológicos. Disponível em: <http://www.snirh.gov.br/hidroweb/>. Acesso em: 13 ago. 2017.

SOARES, D. B. Degradação ambiental no semiárido pernambucano: contribuição ao estudo da desertificação. (Dissertação de Mestrado). Programa de pós-graduação em Desenvolvimento e Meio Ambiente. Pernambuco: PRODEMA/UFPE, 2012.

SOUZA, J. F. de; SILVA, R. M.; SILVA, A. M. Influência do uso e ocupação do solo na temperatura da superfície: o estudo de caso de João Pessoa - PB. Ambient., v. 16, n. 1, p. 21-37, 2016. 
SPINONI, J. et al. World drought frequency, duration, and severity for 1951-2010. Int. Journal Climatol., v. 34, p. 2792-2804, 2014.

SPINONI, J. et al. European drought climatologies and trends based on a multi-indicator approach. Glob. Planet. Change 1, v. 27, p. 50-57. 2015.

TOMASELLA, J.; VIEIRA, R. M. S. P.; BARBOSA, A. A.; RODRIGUEZ, D. A.; OLIVEIRA, M. S.; SESTINI, M. F. Desertification trends in the Northeast of Brazil over the period 2000-2016. International Journal of Applied Earth Observation and Geoinformation. , v.73, p.197 - 206, 2018.

VIEIRA, R. M. S. P. et al. Identifying areas susceptible to desertification in the Brazilian northeast. Solid Earth, v. 6, p. 347-360, 2015.

VIEIRA, R. M. S. P. et al. Land use and land cover map of a semiarid region of Brazil for meteorological and climatic models / Mapa de uso e cobertura da terra do semiárido do Brasil para modelos climáticos e meteorológicos. Revista Brasileira de Meteorologia, v. 28, n. 2, p. 129-138, June 2013.

WAN, Z.; LI, Z. L. A physics-based algorithm for retrieving land-surface emissivity and temperature from EOS/ MODIS data. IEEE Transactions on Geoscience and Remote Sensing. v. 35, n. 4, p. 980-996, 1997.

WANG T.; SUN, J. G.; HAN, H. et al. The relative role of climate change and human activities in the desertification process in Yulin region of northwest China. Environmental Monitoring and Assessment, v. 184, n.12, p. 71657173, 2012.

WANG, X. M.; CHEN, F. H.; DONG, Z. B. The relative role of climatic and human factors in desertification in semiarid China. Global Environmental Change, v. 16, n. 1, p. 48-57, 2006.

XU, D. Y. et al. Multi-scale quantitative assessment of the relative roles of climate change and human activities in desertification - a case study of the Ordos Plateau, China. Journal of Arid Environments, v. 74, n. 4, p. 498-507, 2010.

ZDRULI, P. Land resources of the mediterranean: status, pressures, trends and impacts on future regional development. Land. Degrad. Dev., v. 25, p. 373-384, 2014.

ZHENG, Y. R. et al. Did climate drive ecosystem change and induce desertification in Otindag sandy land, China over the past 40 years? Journal of Arid Environments. v. 64, n. 3, p. 523-541, 2006. 